\title{
The surgical treatment of cervical myelopathy due to spondylosis and disc degeneration
}

\author{
RV JEFFREYS \\ From the Mersey Regional Department of Neurosciences, Walton Hospital, Liverpool, UK
}

SUMMARY A personal prospective study of the surgical treatment of cervical myelopathy due to spondylosis and disc protrusion is presented. One hundred and thirty-seven patients with severe disability have undergone surgery according to a protocol involving both the anterior approach of Cloward and decompressive laminectomy. Fifty-two percent of patients have returned to full employment, $39 \%$ to light employment and $9 \%$ remained disabled though improved from their pre-operative status. The pathenogenesis, natural history and treatment are discussed and reasons advanced to suggest that the disability resulting from severe forms of the condition may be alleviated in the majority of cases.

Cervical spondylosis is widespread throughout the adult population; it has been estimated that, on the basis of changes on plain radiographs of the cervical spine, $50 \%$ of people over the age of 50 years and $75 \%$ over the age of 65 suffer from the disease. It is indeed fortunate that only a small proportion develop the most serious complication of the disease, namely cervical spondylotic myelopathy; however, when this occurs it can lead to devastating and crippling neurological deficit. The treatment of cervical spondylotic myelopathy still is the cause for debate, since the results of treatment vary greatly. The results following decompressive cervical laminectomy have not been strikingly better than non-operative treatment; ${ }^{1}$ hence the anterior approach, initiated independently and contemporaneously by Cloward ${ }^{2}$ and Smith and Robinson ${ }^{3}$ has been favoured latterly.

In an earlier paper ${ }^{4}$ the author reported his results in 63 patients using a prospective protocol in which both the anterior approach of Cloward and decompressive laminectomy were employed; specific criteria were laid down for the appropriate operation; 50 patients underwent a Cloward's operation and 13 a laminectomy, with the result that $80 \%$ of patients were greatly improved. This paper will report the results of a further 74 patients with cervical spondylotic myelopathy, using the same protocol; the ini-

Address for reprint requests: Mr RV Jeffreys, Walton Hospital, Liverpool L9 IAE.

Received 2 April 1985 and in revised form 7 August 1985. Accepted 8 August 1985 tial 63 patients with updated follow up have been included, thus making a total of 137 patients treated consecutively and personally under the same protocol.

\section{Protocol}

Patients with cervical myelopathy were admitted to the Mersey Regional Department of Neurosciences, which is exclusively responsible for the neurosurgical services to a catchment area of 2.9 million people. After plain radiographs of the cervical spine all patients underwent myelography, prior to 1978 with iodophenylate and since then with water soluble agents (metrizamide or iopamidol). Patients with other pathological processes were excluded, and only those patients with evidence of a myelopathy exclusively due to cervical spondylosis or disc degeneration were included.

In every case the myelogram showed major changes, and since all the patients were steadily deteriorating it was felt that surgery was indicated. The aim whenever possible was to decompress the spinal canal by the anterior approach and to reserve the posterior approach (laminectomy) for either widespread or predominantly posterior compression. The more specific indications were as follows:

Cloward's operation To remove any anterior protrusion extending $4 \mathrm{~mm}$ or more beyond the level of the vertebral body, even if this meant multiple level operations. Any protrusion $3 \mathrm{~mm}$ or less was left alone.

Laminectomy (1) Patients with multiple protrusions of $3 \mathrm{~mm}$ or less and with a narrowing of the theco-periosteal diameter in the saggittal plane to less than $12 \mathrm{~mm}$ at one or more levels. (2) Patients with no anterior protrusions but in whom there were multiple posterior indentations, and again with a narrowing of the canal. (3) Patients with a congenitally narrow canal and superimposed narrowing from spondylosis, yet without a major anterior protrusion. 
The neurological details are described later.

By this protocol 108 patients underwent the anterior approach and 29 a laminectomy. Ten patients following an initial Cloward's operation required a second operation, five undergoing a second Cloward's operation and five a decompressive laminectomy.

\section{Operative technique}

\section{Cloward}

The technique used was basically that described by Cloward ${ }^{2}$ with the modifications described by the author in $1979,{ }^{4}$ which reduced the number of tools within the neck, thereby facilitating both vision and operative technique, and of allowing radiology as far distally as the first thoracic veretebra (using image intensification) even in the most kyphotic of patients. The contiguous vertebral bodies were drilled only as far as the posterior cortical plate in order to prevent that most disastrous accident of this operation: drilling into the dura or spinal cord. The depth of the drill was set at $18 \mathrm{~mm}$ in a woman, and $20 \mathrm{~mm}$ in a man, from the anterior surface of the vertebral body. These measurements were arrived at as the result of a series of measurements involving the degree of magnification on 20 myelograms and which showed that the magnification ranged from $1 \cdot 16-1 \cdot 6$, thus plainly indicating that the depth of drilling based upon measurements from radiographs was unreliable for an individual patient.

Once drilling was complete the cortical plate was pared down with mastoid curettes and punches before removing the compressive lesion which could be osteophyte, disc or both (see later). The dissection was best done between vertebral body and posterior longitudinal ligament thereby reducing bleeding to a minimum; however, if there was a disc protrusion and a hole was seen in the posterior ligament this was enlarged in order to remove the free fragment of disc which usually under these circumstances lay within the canal. The canal was thoroughly decompressed both centrally and laterally so that by the end the posterior ligament moved anteriorly against the vertebral bodies. Dowels $(12 \mathrm{~mm})$ were cut from the right iliac crest and gently tapped into place and countersunk for 1-2 mm.

Postoperatively each patient was fitted with a firm cervical collar which was worn for 3-4 weeks and then discarded. Patients were mobilised on the first postoperative day and given physiotherapy and occupational therapy for 10-12 days and were then discharged home if they were able to go up/down stairs unaided, or if unable to so do they were transferred to the referring district general hospital before going home. Eighty-five per cent of patients were able to go straight home, and the rest were transferred back to the referring hospital though none of these remained in hospital more than 1 month after surgery.

\section{Cervical laminectomy}

The operation was performed in the sitting position; this had the combined advantages of both slightly flexing the cervical spine thereby putting the posterior cervical muscles on the stretch so that they could be parted easily, and of allowing blood to run away from the operating area. This position is greatly superior to the prone position in all respects except one: the risk of air embolism. By using an ultrasonic micro- phone placed over the precordium very small amounts of air $(1 \mathrm{ml})$ can be detected early so that the surgeon is immediately aware of the potential hazard and can take the appropriate steps to deal with the offending vein. A laminectomy from $\mathrm{C} 3$ to either $\mathrm{C} 6$ or $\mathrm{C} 7$ was carried out in every case, even if the myelogram had shown a more localised lesion, since it was felt it was better to allow the dural sac, with the contained spinal cord, to bulge gently backwards over a long segment rather than bulge acutely through a small decompression. The dura was not opened.

Post-operative management was the same as that following a Cloward's operation, except that a cervical collar was deemed unnecessary.

\section{Results}

For reasons of clarity the whole series will be discussed together until the time of operation, then the patients undergoing Cloward's operations will be presented separately from those undergoing laminectomy. At the end the two groups will be rejoined and the final outcome of the whole series will be reviewed.

\section{Pre-operative details}

The whole series comprises 137 patients treated between January 1975 and June 1984 (9.5 years). All patients were admitted under the care of the author who performed 127 of the operations and supervised trainees in the other 10 . The mean age was 57.01 years (range 29-80), and the sex ratio in favour of men was 2:1 (93:44). The duration of symptoms is shown in the fig, from which it can be seen that $53.4 \%$ had a history of 12 months or less, and $46.6 \%$ a history in excess of 12 months. All patients were asked two

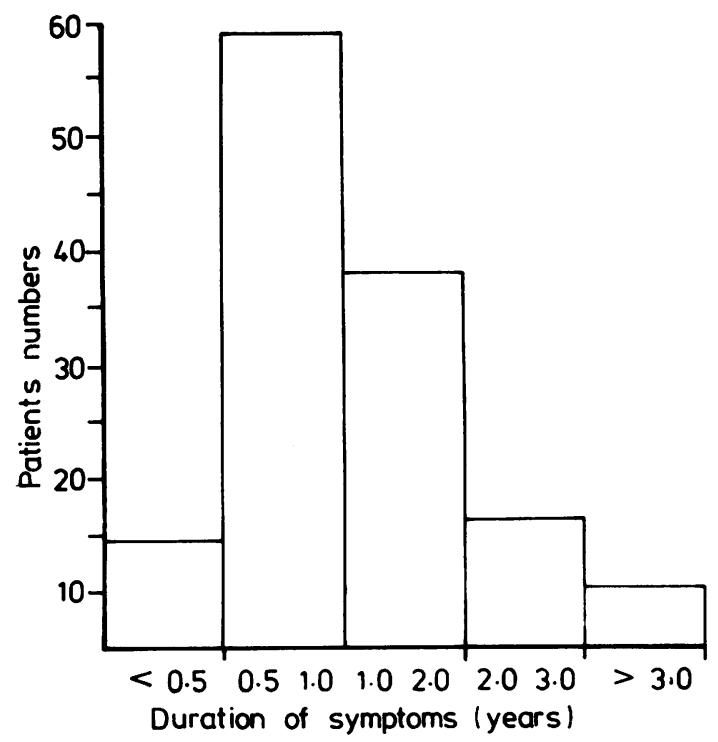


Table 1 Functional grading

1 Totally disabled and dependent on others for daily living.

2 Disabled living at home but capable of daily living for eating and personal hygiene

3 Capable of light employment; in case of age retired capable of cleaning and driving car and light chores in the house and of doing the shopping.

4 Capable of full employment; in case of age retired capable of repairing car, painting and decorating house, climbing ladders and of walking up to 4 miles at a time (or such activities as playing golf).

questions regarding the influence of trauma: whether or not they had suffered an injury to the neck in the past, and whether or not their present symptoms had been precipitated by a neck injury. Only an injury in the past had been experienced by $10.94 \%, 8.0 \%$ had suffered a precipitating injury only and $16.0 \%$ had suffered both a past and a precipitating injury.

The function of patients was graded on a scale (table 1) which was used both before and after operation; preoperatively $55.47 \%$ were graded 1 , that is to say were so handicapped that they were dependent on others for daily living, and $44.52 \%$ were graded 2 , that is handicapped in such a way that they could manage daily living at home but little else.

The overall neurological picture fell into one of four main categories ( $\%$ of cases in brackets):

(1) Ataxia and spasticity of the legs together with loss of fine function of the hands; subjective loss of feeling in the fingers but little or no objective sensory deficit $(30.65 \%)$.

(2) Ataxia and spasticity of the legs together with wasting of the arms; loss of fine feeling in the fingers but often little or nor objective sensory deficit $(42 \cdot 33 \%)$.

(3) Partial Brown-Sequard syndrome (6.56\%).

(4) A motor/sensory spastic tetraparesis with varying proportions of motor:sensory signs $(20.43 \%)$.

Other diseases, either before or after operation were suffered by $46.7 \%$, the most common of which were diabetes mellitus $(4.4 \%)$, severe chronic obstructive airway disease $(3.6 \%)$, carcinoma of the bronchus $(2.9 \%)$ and myocardial infarction $(2 \cdot 9 \%)$. All the cases of bronchial carcinoma occurred postoperatively but none of the other disease processes affected the treatment of the myelopathy. In particular it should be stressed that those cases with pul- monary dysfunction came through their surgery easily and their postoperative pulmonary function was much improved.

Pre-operative radiological investigation consisted in every case of plain radiographs of the cervical spine and myelography. In $81.75 \%$ the plain radiographs revealed the typical features of cervical spondylosis, and $15.3 \%$ had normal radiographs. In $2.95 \%$ a subluxation was present, in addition to spondylotic changes, but in no case did this change in radiographs taken in flexion. Myelography was performed with iodophenylate prior to $1978(37.95 \%)$, and since then with the water-soluble agents metrizamide and iopamidol $(62.05 \%)$. The myelographic changes could be subdivided into one of four groups $(\%$ of cases in brackets):

(1) Anterior indentation/s (59.12\%).

(2) Posterior indentation/s (4.37\%).

(3) Partial block, in which the passage of dye was held up in one position by an anterior or posterior indentation, but would pass the obstruction when the position of the neck was changed $(24.0 \%)$.

(4) Complete block in any position (12.4\%).

Of the 137 patients 127 underwent one operation only: 98 Cloward's procedure and 29 laminectomy. Ten patients underwent two operations, five had a second Cloward's and five after an initial Cloward's operation had a laminectomy; since these patients all underwent a Cloward's operation as the initial procedure their results are included in the overall Cloward's group; thus the results for 108 patients undergoing Cloward's operation and 29 laminectomy are now discussed separately.

\section{Cloward's operation}

The protocol and operative technique for patients undergoing Cloward's operation have already been described. The mean age was $56 \cdot 3$ years and the sex ratio in favour of men $2 \cdot 2: 1$. The protocol resulted in 108 patients having 140 disc spaces dealt with: 77 $(71 \cdot 3 \%)$ having one space operation, $30(27 \cdot 8 \%)$ two spaces and $1(0.9 \%)$ three spaces; the $4 / 5$ and $5 / 6$ levels were the most frequent. At each operation a record was made of the nature of the compressive lesion/s. In no case in whom more than one space was explored were there different lesions at different spaces and the

Table 2 Cloward results

\begin{tabular}{llcc}
\hline Functional grading & $\%$ Patients with Cloward's operation & \\
\cline { 2 - 4 } Grade & Pre-op \% & 4 mths post-op \% & 6 mths post-op \% \\
\hline 4 & $0 \cdot 0$ & $8 \cdot 4$ & $54 \cdot 6$ \\
3 & $0 \cdot 0$ & $72 \cdot 2$ & $36 \cdot 1$ \\
2 & $50 \cdot 0$ & $18 \cdot 5$ & $9 \cdot 3$ \\
1 & $50 \cdot 0$ & 0.9 & $0 \cdot 0$ \\
\hline
\end{tabular}


Table 3 Complications of Cloward's operation

\begin{tabular}{ll}
\hline Painful hands & 5 \\
Worse immediately post-op & 2 \\
Donor site infection & 3 \\
Tear in vertebral artery & 1 \\
Pneumonia & 1 \\
Epilepsy & 1 \\
Psychosis & 1 \\
Bleeding duodenal ulcer & 1 \\
Total & $15(14 \%)$ \\
\hline
\end{tabular}

figures therefore relate to patients rather than disc spaces: (1) osteophyte $(38.9 \%)$, (2) disc $(24.0 \%)$, (3) osteophyte and disc $(37 \cdot 1 \%)$.

As a result of these operative findings a correlation with the pre-operative plain radiographs was sought. Whereas as one would have predicted $95 \%$ of the operative osteophyte group and $87 \%$ of the mixed group showed spondylotic changes on plain radiographs, it is important to note that only $50 \%$ of the group in whom a pure disc protrusion was found to be the compressive lesion showed spondylotic radiographic changes.

The functional results of treatment are shown in table 2. Patients attended for follow-up at approximately 6 weeks, 4 months and 6 months after discharge from hospital. Those who had reached grade 4 were then discharged from the clinic but they were encouraged to return if they developed any further problems no matter how minor. Since the neurosurgical department is the only one in Mersey and North Wales and since patient movement out of the area is minimal (for economic reasons), there are good grounds for believing that patients would have returned if they had developed any problems. In fact the vast majority of patients were so grateful that they kept in touch on an informal basis. All other patients were seen at yearly intervals.

It is interesting to note that although $72.2 \%$ had improved to grade 3 by the end of four months, by the end of six months many had improved further so that they were capable of returning to their old occupation, or in the case of the retired ( $>60$ years) were able to perform tasks in the home, which if they had been at work would have qualified them as manual workers. Overall $54.6 \%$ were capable of performing thus, and $36.1 \%$ were capable of work in a lighter role. Only $9.25 \%$ were left with function incapable of work, and no patients were worse off as a result of surgery.

Complications fell into one of three main categories: as a result of surgical technique, or delayed perhaps as a result of incorrect decision taking or unrelated (table 3). Two complications occurred during the operation; in one case a small hole was made in an atheromatous vertebral artery which had eroded through to the spinal canal; the decompressive part of the operation was completed and the dowel was inserted and this stopped the bleeding; after the operation the patient made a full and uneventful recovery. In the other case the curette slipped and hit the spinal cord; there was no doubt that postoperatively the patient was worse for one week though ultimately he 8 made a full recovery.

The final outcome in terms of function was $\overrightarrow{0}$ unaffected by the myelographic finding but did appear to be adversely affected by the pre-operative

Table 4 Effect of five clinical variables on outcome in 137 cases

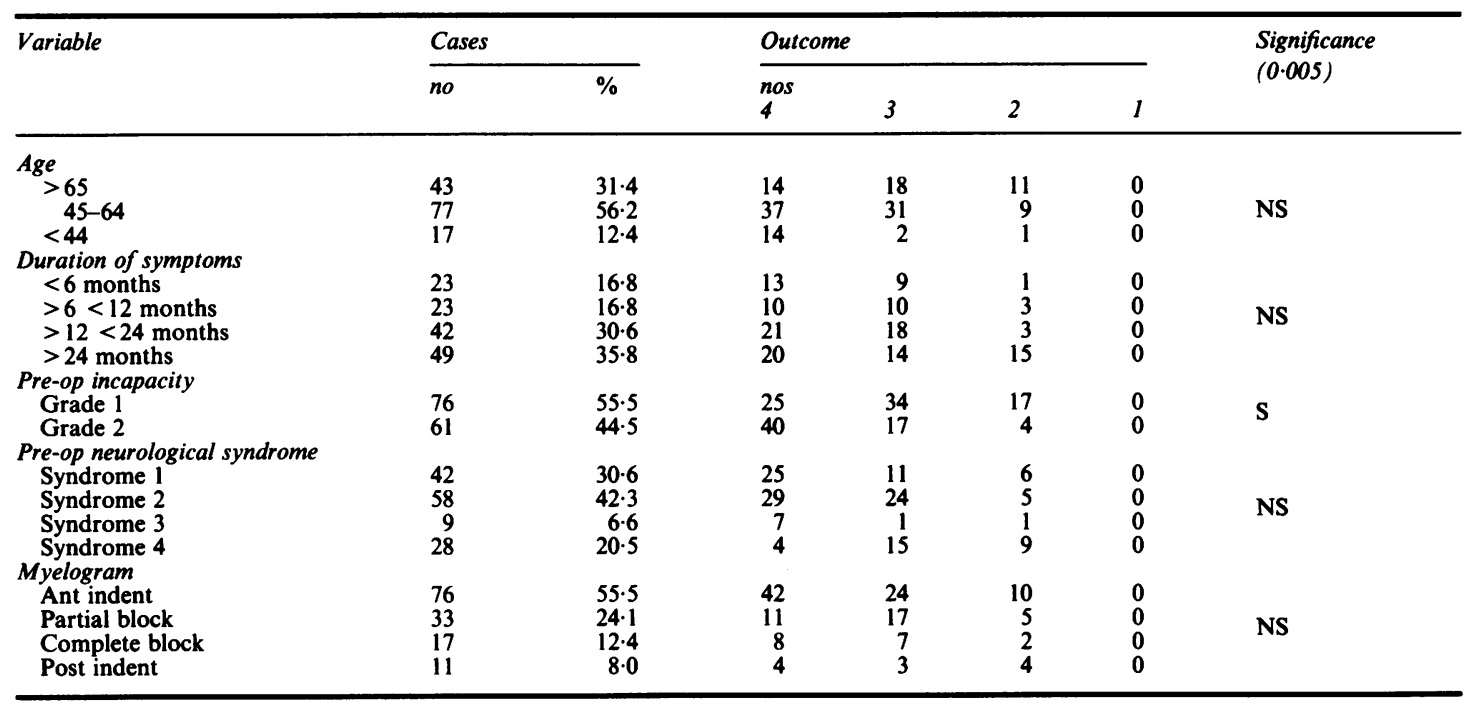


Table 5 Neurological vs functional outcome (\% patients)

\begin{tabular}{llcrrr}
\hline Neurological grading & \multicolumn{2}{l}{ Functional grading 6 mihs } & Total \\
\cline { 2 - 5 } & 4 & 3 & 2 & 1 \\
\hline 3 & $47 \cdot 4$ & $4 \cdot 4$ & 0 & 0 & $51 \cdot 8$ \\
2 & 0 & $32 \cdot 2$ & $7 \cdot 3$ & 0 & $39 \cdot 5$ \\
1 & 0 & $0 \cdot 7$ & $8 \cdot 0$ & 0 & $100 \cdot 0$ \\
Total & $47 \cdot 4$ & $37 \cdot 31$ & $15 \cdot 3$ & 0 \\
\hline
\end{tabular}

See text for neurological grading.

findings of a tetraparesis and grade 1 incapacity and favourably affected by the findings of a disc at surgery and grade 2 pre-operative incapacity.

\section{Cervical laminectomy}

The average age was 59.6 years and the sex ratio was $1 \cdot 6: 1$ in favour of men, and thus was not significantly different from the Cloward group, although there was a difference in the preoperative incapacity, $76 \%$ being grade 1 and $24 \%$ being grade 2 (cf $55 \%$ and $53 \%$ respectively for the Cloward group). The follow-up was identical to the Cloward group. After operation, at 6 months $20.7 \%$ achieved grade 4 function, $41.4 \%$ grade 3 and $37.9 \%$ grade 2 . No patient was worse as a result of surgery. There were two complications $(6 \%)$ of a minor nature.

\section{Overall results}

Age appeared to have some bearing on outcome, although results of the three age groups were not statistically significant (table 4). However, it must first be pointed out that those patients over 65 years were more disabled than those aged 45-64, who in turn were more disabled than those aged less than 44 years (table 5). The duration of symptom's had no bearing on outcome, neither did the pre-operative neurological condition. The severity of pre-operative incapacity had a statistically significant bearing on outcome with grade 1 patients faring less well than grade 2 (table 5).

Overall $65(47 \cdot 4 \%)$ patients were improved to grade 4 , so that they were capable of full activities such as their old job or a full and active retirement if over the age of 60 years. Fifty-one $(37 \cdot 3 \%)$ reached grade 3 so that they were capable of lighter employment or a reasonably rewarding retirement. Twentyone $(15.3 \%)$ were left at grade 2 capable of independent existence both in the home and its immediate environs, but incapable of manual employment.

These results were assessed at 6-9 months after operation. However, continuing observation showed that nine $(6.6 \%)$ patients deteriorated later. All of these were re-investigated, and in five further compressions were found that responded to a subsequent second Cloward's operation; these patients regained their original post-operative level achieved after the first operation. In the remaining four patients the repeat myelogram showed no compression and the impression was gained that they were suffering from demyelination. If one reassesses the overall figures then the grade 4 result is still $65(47 \cdot 4 \%)$ but grade 3 becomes $47(34.3 \%)$ grade $2,23(16.8 \%)$ and grade 1 , $2(1 \cdot 5 \%)$.

Although the functional result is all important to the patient, the objective neurological result is also of interest to the surgeon, and patients were graded after operation on the following scale:

Grade 3 Normal, or normal in every way other than increased tendon reflexes.

Grade 2 Mild myelopathic signs with slight weakness and ataxia when challenged though capable of normal gait when not under pressure. Slight loss of fine movements of the hands.

Grade 1 Clear cut signs of myelopathy.

From table 5 it will be seen that there is a good correlation between what the patient was able to do and what the observer found on neurological examination in the outpatient clinic. The presence of increased tendon jerks in the absence of spasticity did not seem to affect function. The other interesting fact to emerge was that ataxia could be demonstrated on walking in the absence of obvious posterior column loss.

\section{Discussion}

The three main points for any discussion on cervical spondylotic myelopathy relate to pathenogenesis and aetiology, natural history and treatment.

Aetiology Such a discussion can be divided into two: first the aetiology of cervical spondylosis and its relationship to disc degeneration; and second, given the high frequency of cervical spondylosis in the population over 45 years of age, what causes a minority to develop the major complication of a myelopathy. Regarding the latter various theories have been advanced to explain cervical spondylotic myelopathy, and to some extent these interrelate; these are concerned with cord compression, ischaemia, and cord and root traction. It is important to define these terms 
accurately. By ischaemia is meant a reduction in arterial blood supply to the cord independent of cord compression. Since occlusion of the anterior spinal artery is very rare in cervical spondylotic myelopathy ${ }^{5}$ proponents of this theory implicate either the vertebral artery itself or the reinforcing radicular arteries. Hughes ${ }^{6}$ points out that he failed to demonstrate any occlusion or stenosis in his necropsy studies of patients with cervical spondylotic myelopathy. Frykholm ${ }^{7}$ emphasised the importance of root-sleeve fibrosis and it was thought possible that the reinforcing radicular arteries might be involved also; however as Nurick ${ }^{8}$ points out that with such a theory one might expect patients with radicular lesions to develop further a myelopathy. Although a small number of patients do so the vast majority do not, and for this reason this theory has to be rejected. Adams and Logue ${ }^{9}$ felt that traction of the spinal cord against osteophytes projecting from the posterior surface of the vertebral bodies is more important, so that they felt the rationale of treatment should be to reduce or eliminate such movement and they also felt that compression could not explain every case and the traction theory could explain some. Barnes and Saunders ${ }^{10}$ found that patients who deteriorated were likely to have more mobility of the cervical spine than those who remained functionally static. If mobility were a major causative factor one would expect a significant proportion of patients to be benefited by wearing a firm cervical collar; in this present series many patients had tried such treatment and to no avail.

Nurick ${ }^{8}$ discussed the pathenogenesis of the spinal cord disorder associated with cervical spondylosis, and came to four major conclusions, based on an assessment of 135 patients with myelopathy: (1) The constitutional and acquired sagittal diameters of the cervical canal are narrower in patients with cervical spondylotic myelopathy than in others, and are narrowest in those most severely affected. (2) Subluxation is also more frequent in those with greater disability. (3) The clinical, radiological and pathological data together suggest that the changes in the spinal cord are secondary to compression. (4) Ischaemia of the cord occurring independently of compression does not explain the development of cervical spondylotic myelopathy. In a companion paper $^{11} 91$ patients undergoing treatment were reviewed and it is clear that there is a major difference between the series in that $70 \%$ of Nurick's patients were mildly affected (= grade 4 and 3 in this series) whereas in this present series $100 \%$ of patients were severely affected (grades 1 and 2) with progressive disability, and thus this present series is therefore selected whereas in Nurick's series ${ }^{811}$ there was a spectrum of disability with a predominance of the less severely affected; hence he concluded that cervical spondylotic myelopathy is a generally benign disorder.

The role of trauma in the development of cervical spondylotic myelopathy is an interesting one; in this series $10.94 \%$ gave a history of a neck injury in the past, $8.0 \%$ experienced a neck injury which precipitated a neurological decline and $16.0 \%$ gave a history of both, that is approximately $44 \%$ experienced a neck injury at some time. Nurick does not mention a past history of neck injury although he found that $14 \%$ experienced an injury in the month prior to admission and which had frequently precipitated the final decline. Although there is no proof it would seem reasonable to infer that the past neck injury may have contributed to the degenerative disease and the precipitating injury in some way further compromised a spinal cord already at risk.

Whilst accepting that a constitutionally narrow canal may have a major bearing on the production of cervical spondylotic myelopathy, it is also clear that even patients who develop cervical spondylotic myelopathy later in life are perfectly able to live normal and neurologically asymptomatic lives until there is a superadded narrowing from various tissues of the vertebral column. In cervical spondylotic myelopathy the nature of the compressing material can be anterior to the cord when it may be osteophyte, disc or combinations of these and/or posterior when it will be ligamentum flavum or lamina. In this present series, based upon the myelographic and operative findings, it was found that the major compressive lesion was anterior to the cord in $103(75.2 \%)$ patients, posterior in $29(21.2 \%)$ and both anterior and posterior in 5 $(3 \cdot 6 \%)$. The nature of the anteriorly compressing lesion was osteophyte alone in $39 \%$, disc alone in $24 \%$ and combinations of osteophyte and disc in $37 \%$. It is important to point out that even in those patients in whom disc material was found to be the major compressive agent $50 \%$ of their plain radiographs showed the changes of spondylosis. Whereas it would probably be universally accepted that in a cervical spine that had undergone spondylotic changes the intervertebral disc will be both degenerate and atrophic, it is interesting to note that in a large number of patients there was sufficient disc material to play an important role in compression of the cord in addition to that of osteophytic protrusion.

The role of subluxation in the evolution of cervical spondylotic myelopathy remains problematical. In this series subluxation was only found in $2.95 \%$ of patients, whereas Nurick found that not only did this radiological sign occur in $28.7 \%$ but that it tended to be associated with increasing disability. One possible explanation of this discrepancy may be that in this present series the patients were more disabled and 
possibly had more advanced spondylosis, so that their cervical spines were more rigid and less able to dislocate than those in Nurick's series. In addition there is the finding by Adams and Logue, ${ }^{12}$ who found that those who improved most after decompressive laminectomy were those who had the least movement of the cervical spine.

Finally there is the evidence from this present series that a large majority of patients improved greatly once the compressive agent had been removed and most of these patients had mobile cervical spines afterwards. The author feels that although there is no absolute proof the theory most likely to explain a myelopathy is compression of the spinal cord provided that one also allows that in any compression there must be an element of local ischaemia in the cord at the site of compression, caused by the compressing agent "squashing" the vascular bed at that point.

\section{Natural history}

As Lunsford et al ${ }^{13}$ have reported, the symptoms of cervical spondylotic myelopathy may be insidious, progressive and unrelenting or intermittent and associated with remissions. Moreover as Phillips ${ }^{14}$ mentions, a number of studies by physicians ${ }^{111516}$ have emphasised that often cervical spondylotic myelopathy is a benign condition, which has clearly not been the finding in this present series where all the patients were seriously disabled. Whilst there is undoubted variability of the clinical course the discrepancy between the findings of neurosurgeons and others probably relate to the mode of referral and the differing attitudes to the various types of treatment that are available.

Nurick ${ }^{11}$ found that the single feature most often associated with deterioration was the age of the patient at presentation. This present author did not refuse any patient on the grounds of age and yet the maximal age incidence was 50-70 years; the fall-off during later decades is probably a reflection not only of a population less numerous but also the reluctance of geriatricians to refer such patients. However the proportion of patients in grade 1 and grade 2 at referral steadily rose with increasing age and this finding very much agrees with that of Nurick, although the duration of symptoms did not alter with age. Moreover one has to consider physiological age as opposed to chronological age. In this present series $46.7 \%$ patients were found to have suffered or be suffering from another unrelated disease, a finding which would seem to indicate that as a group patients with cervical spondylotic myelopathy are far from being generally healthy.

Few authors state the duration of symptoms at time of referral; in Nurick's ${ }^{11}$ series the mean duration was 27.8 months and in Barnes and Saunders ${ }^{10}$ it was 14 months as compared to 21.48 months in this present series. However the mean duration obscures the fact that $53 \%$ patients experienced their symptoms for less than 12 months. Although almost certainly in this present series cervical spondylosis has been present in the patients for a number of preceding years it is also quite clear that in half the patients once neurological symptoms appeared they progressed quite rapidly; whether or not the decline would have arrested as suggested by some authors, is not known since the author was not prepared to run the risk of yet further decline in patients' functions.

\section{Treatment}

An essential prerequisite to a discussion on the results of management of cervical spondylotic myelopathy is an accurate diagnosis. Since cervical spondylosis is so common in the population over the age of 45 years it cannot be assumed that a patient with myelopathy and plain radiograph changes of spondylosis necessarily has cervical spondylotic myelopathy, particularly so since Campbell and Phillips ${ }^{17}$ found that $17 \%$ patients presumed to have cervical spondylotic myelopathy were subsequently shown to have another disease. At the present time there are only two radiographic ways to examine the vertebral column and spinal cord in length: contrast myelography and NMR. The latter may well become the investigation of choice but for the present, owing to financial restrictions, myelography will continue to be the method generally used. Once other structural lesions have been excluded and the compression due to osteophytes and/or disc confirmed there are three other diseases to be excluded: motor neuron disease, subacute combined degeneration of the cord and demyelination. The former two can usually be diagnosed by the standard methods. Demyelination may prove difficult to diagnose and in this present series was thought to occur in four patients all of whom were initially improving following surgery only to deteriorate later with other neurological deficits. There was only one patient who later developed clear evidence of motor neuron disease.

The treatment of cervical spondylotic myelopathy still remains a difficult and contentious problem. On the basis that a relatively mobile cervical spondylotic cervical spine is more likely to lead to advancing myelopathy ${ }^{910}$ reduction of mobility by a firm cervical collar has been advocated; Barnes and Saunders ${ }^{10}$ point out the results are variable though this may relate to the compliance rate. In this present series many of the patients had tried this treatment and yet continued to deteriorate. Perhaps in the patient with early and slight myelopathy there is a place for a trial 
of such treatment, but in the patient with advancing myelopathy then surgery is indicated. ${ }^{118}$

The two surgical approaches are decompressive laminectomy and the anterior approach. ${ }^{23} \mathrm{~A}$ review of the published reports of laminectomy ${ }^{1}$ showed that $56 \%$ patients were improved following operation, $25 \%$ were unchanged and $19 \%$ were worse (the corresponding figures for non-operative treatment were $40 \%, 36 \%$ and $24 \%$ ). These figures do not show much superiority for laminectomy, though with more detailed analysis it can be shown that laminectomy produces better results in those less severely disabled and who have a shorter history. Phillips ${ }^{14}$ found that in a series of 102 patients with cervical spondylotic myelopathy, treated by either a minerva collar, laminectomy or Cloward's operation, the best results were obtained by Cloward's operation with $73 \%$ showing sustained improvement and $58 \%$ returning to and remaining at work. Similar results were reported by Dereymaeker et al. ${ }^{19}$ In this present series $82 \%$ were at work or capable of work, $17 \%$ were independent at home and only $1.2 \%$ were dependent on others at home. However there have been disappointing results reported; Galera and Tovi ${ }^{20}$ noted that only $39 \%$ improved after anterior surgery and in a more recent series $^{20}$ only $50 \%$ did so.

There are several possible reasons for these differences. Firstly careful selection of patients is essential, ${ }^{1}$ since it is important to exclude patients with other causes for their myelopathy. Secondly there has to be a rational approach to the problem of cervical spondylotic myelopathy. As Nurick ${ }^{1}$ stated "if cervical spondylotic myelopathy is considered to be a condition of compression of the spinal cord caused by a narrow canal, with superimposed changes due to spondylosis, the management can be considered rationally". It can be argued that no evidence exists for the superiority of one surgical method over the other; however, various authors ${ }^{1412}$ have stated that such an approach is restrictive and both the anterior and the posterior surgical approaches have a place. Nurick ${ }^{1}$ advocated that the anterior approach would be best for disc or osteophyte protrusion at one or two levels, and laminectomy would be the procedure of choice for a narrow canal with perhaps multiple protrusions each one of a more minor nature although together contributing to the overall compression. In an earlier report the present author ${ }^{4}$ concurred with these views and added to it simply by putting a value to the height of the protrusion (namely $4 \mathrm{~mm}$ ) which required anterior decompression.

Although it cannot be proved there remains a suspicion that in some other reports authors when they use the term "anterior fusion" do in fact mean just this. In the opinion of this author a fusion alone is insufficient and that what really matters is a thorough decompression of the spinal cord; it is essential that by the end of decompression of each disc space the posterior longitudinal ligament moves forwards against the vertebral bodies. The question of whether or not to fuse the disc space with a bone graft remains open; O'Laoire and Thomas ${ }^{21}$ did not do so in their series of 26 patients with cervical disc protrusion. This author would be prepared to accept that a soft disc can be excised by what is essentially a soft tissue operation, but doubts whether osteophytes protruding into the spinal cord can be adequately removed through such a small approach. Having performed a Cloward's operation he feels it wise to "fill in the hole" with a graft, since this also has the merits of both instantly fixing the space and eliminating movement, and of helping to maintain the normal lordosis of the cervical spine. Whether or not in the very long term a fusion with a graft is superior to allowing the disc space to collapse is not known at the present time.

An important point regarding management of cervical spondylotic myelopathy revolves around the dangers of surgery against those of non-operative management. An imposing list of potential complications, some of which can be catastrophic, can be made. ${ }^{1314}$ However as this present series demonstrates the complication rate can be kept to the low overall rate of approximately $1 \%$, even in severely neurologically handicapped patients many of whom were suffering from other major diseases. Since in this series all the patients were deteriorating with nonoperative treatment it would seem quite clear that surgery had a lot to offer for at least the short and medium terms; the long-term of 10-20 years is not known in this present series.

Therefore, in conclusion it would seem that with a protocol involving specific indications for both the anterior and posterior approaches many patients with cervical spondylotic myelopathy can be helped back to an active existence. It is to be hoped that some of the gloom surrounding this condition can be lifted.

\section{References}

${ }^{1}$ Nurick S. Cervical spondylosis and the spinal cord. $\mathrm{Br} J$ Hosp Med 1975;13:668-76.

${ }^{2}$ Cloward RB. The anterior approach for removal of ruptured cervical discs. J Neurosurg 1958;15:602-14.

${ }^{3}$ Smith GW, Robinson RA. The treatment of certain cervical spine disorders by anterior removal of the intervertebral disc and inter-body fusion. $J$ Bone Joint Surg 1958;40(A):607-24.

${ }^{4}$ Jeffreys RV. The surgical treatment of cervical spondylotic myelopathy. Acta Neurochirurgica 1979;47:293-305.

${ }^{5}$ Hughes JT, Brownell B. Cervical spondylosis complicated 
by anterior spinal artery thrombosis. Neurology (Minneap) 1964;14:1073-7.

${ }^{6}$ Hughes JT. Pathology of the Spinal Cord. 2nd edition. London: Lloyd-Luke, 1978.

${ }^{7}$ Frykholm R. Cervical nerve root compression resulting from disc degeneration and root-sleeve fibrosis. Acta Chir Scand 1951;suppl 160.

${ }^{8}$ Nurick S. The pathenogenesis of the spinal cord disorder associated with cervical spondylosis. Brain 1972; 95:87-100.

${ }^{9}$ Adams CBT, Logue V. Studies in cervical spondylotic myelopathy. 2 . The movement and contour of the spine in relation to the neural complications of cervical spondylosis. Brain 1971;94:569-86.

${ }^{10}$ Barnes MP, Saunders M. The effect of cervical mobility on the natural history of cervical spondylotic myelopathy. J Neurol Neurosurg Psychiatry 1984;47:17-20.

${ }^{11}$ Nurick $S$. The natural history and the results of surgical treatment of the spinal cord disorder associated with cervical spondylosis. Brain 1972;95:101-8.

${ }^{12}$ Adams CBT, Logue V. Studies in cervical spondylotic myelopathy. 3. Some functional effects of operations for cervical spondylotic myelopathy. Brain 1971; 94:587-94.

${ }^{13}$ Lunsford LD, Bissonette PAC, Zorub DS. Anterior surgery for cervical disc disease. 2. Treatment of cervical spondylotic myelopathy in 32 cases. $J$ Neurosurg 1980;53:12-19.

${ }^{14}$ Phillips DG. Surgical treatment of myelopathy with cervical spondylosis. J Neurol Neurosurg Psychiatry 1973; 36:879-84.

${ }^{15}$ Bradshaw P. Some aspects of cervical spondylosis. $Q J$ Med 1957;26:177-208.

${ }^{16}$ Lees F, Turner JWAT. Natural history and prognosis of cervical spondylosis. Br Med J 1963;2:1607-10.

${ }^{17}$ Campbell AMG, Phillips DG. Cervical disc lesions with neurological disorder. $\mathrm{Br}$ Med J 1960;2:481-5.

${ }^{18}$ Editorial. Management of cervical spondylotic myelopathy. Lancet 1984;2:1058.

${ }^{19}$ Dereymaeker A, Ghosez JP, Henkes R. Le traitement chirurgical de la discopathie cervicale. Neurochirurgie 1963;9:13-20.

${ }^{20}$ Galera GR, Tovi D. Anterior disc excision with interbody fusion in cervical spondylotic myelopathy and rhizopathy. J Neurosurg 1968;28:305-10.

${ }^{21}$ O'Laoire SA, Thomas DGT. Spinal cord compression due to prolapse of cervical intervertebral disc (herniation of nucleus pulposus). Treatment in 26 cases by discectomy without interbody fusion. $J$ Neurosurg 1983;59:847-53. 